\title{
ON $h$-LOCAL INTEGRAL DOMAINS
}

\author{
BY \\ WILLY BRANDAL
}

\begin{abstract}
Related to the question of determining the integral domains with the property that finitely generated modules are a direct sum of cyclic submodules is the question of determining when an integral domain is $h$-local, especially for Bezout domains. Presented are ten equivalent conditions for a Prüfer domain with two maximal ideals not to be $h$-local. If $R$ is an integral domain with quotient field $Q$, if every maximal ideal of $R$ is not contained in the union of the rest of the maximal ideals of $R$, and if $Q / R$ is an injective $R$-module, then $R$ is $h$-local; and if in addition $R$ is a Bezout domain, then every finitely generated $R$-module is a direct sum of cyclic submodules. In particular if $R$ is a semilocal Prüfer domain with $Q / R$ an injective $R$-module, then every finitely generated $R$ module is a direct sum of cyclic submodules.
\end{abstract}

In [8] E. Matlis defines an integral domain $R$ to be $h$-local if every nonzero ideal of $R$ is contained in only a finite number of maximal ideals and if every nonzero prime ideal of $R$ is contained in only one maximal ideal. It is an open question whether an integral domain is $h$-local if it has the property that all finitely generated modules are a direct sum of cyclic submodules. If we had an affirmative answer to this question, then using the results of [1] we would have a generalized fundamental theorem of Abelian groups, i.e., we would have a characterization of the integral domains with the property that every finitely generated module is a direct sum of cyclic submodules.

All rings will be commutative with identity and all modules will be unitary modules. $R$ will always denote a ring and $\Omega$ will denote the set of maximal ideals of $R$. If $A$ is a module, then $A^{*}$ will denote the nonzero elements of $A$. If $R$ happens to be an integral domain, then $Q$ will denote the field of fractions of $R$ and $K$ will denote the $R$-module $Q / R$. If $A$ is an $R$-module, the $R$-topology on $A$ is the topology with the submodules $r A, r \in R^{*}$, being a subbase for the open neighborhoods of 0 in $A$. If $R$ is an integral domain, then $H$ will denote the completion of $R$ in the $R$-topology. $H$ is a ring and $H \cong \operatorname{proj} \lim _{r \in R^{*}} R / R r \cong$

Received by the editors January $25,1974$.

AMS (MOS) subject classifications (1970). Primary 13G05, 13C05, 13F05; Secondary 13C10, 18G05, 06A60.

Key words and phrases. Integral domain, $h$-local domain, Bezout domain, Prüfer domain, finitely generated module, injective module. 
$\operatorname{Hom}_{R}(K, K)$. See [8] or [9] for a discussion of $H$ and the $R$-topology.

A partition of a set $X$ is a finite set $\left\{X_{1}, X_{2}, \cdots, X_{n}\right\}$ of subsets of $X$ such that $X=\bigcup_{i=1}^{n} X_{i}$ and $X_{i} \cap X_{j}=\varnothing$ if $i \neq j$. The partition is said to be nontrivial if $X_{i} \neq \varnothing$ for all $i$. If $I$ is an ideal of $R$, let $\Omega(I)$ denote $\{M \in \Omega: I \subset M\}$.

As a well-known converse of the Chinese Remainder Theorem, we have that if $I, I_{1}, I_{2}, \cdots, I_{n}$ are ideals of $R$ and $R / I \cong \bigoplus \Sigma_{i=1}^{n} R / I_{i}$, then $I=\bigcap_{i=1}^{n} I_{i}$ and $I_{i}+I_{j}=R$ if $i \neq j$, i.e., $\left\{I_{1}, I_{2}, \cdots, I_{n}\right\}$ is pairwise comaximal. As an immediate corollary, if $R / I \cong \bigoplus \Sigma_{i=1}^{n} R / I_{i}$, then $\left\{\Omega\left(I_{1}\right), \Omega\left(I_{2}\right), \cdots, \Omega\left(I_{n}\right)\right\}$ forms a partition of $\Omega(I)$.

E. Matlis has proved that for an integral domain $R, R$ is $h$-local if and only if one of the following equivalent conditions holds: (1) $K \cong \bigoplus \Sigma_{M \in \Omega} K_{M}$, (2) $T \cong \bigoplus \Sigma_{M \in \Omega} T_{M}$ for every torsion $R$-module $T$, or (3) $H \cong \Pi_{M \in \Omega} H(M)$ where $H(M)$ is the completion of $R_{M}$ in the $R_{M}$-topology [9, Theorem 22]. As an improvement of (2) we have the following.

Proposition 1. An integral domain $R$ is $h$-local if and only if $T \cong$ $\bigoplus \Sigma_{M \in \Omega} T_{M}$ for every cyclic torsion $R$-module $T$.

Proof. We only need to prove one implication. Suppose every cyclic torsion module decomposes as indicated, and let $I$ be a nonzero ideal of $R$. Then

$$
R / I \cong \bigoplus \sum_{M \in \Omega}(R / I)_{M} \cong \bigoplus \sum_{M \in \Omega} R_{M} / I_{M}
$$

$M \in \Omega(I)$ implies $R_{M} / I_{M} \neq\{0\} . R / I$ cannot be an infinite direct sum of nonzero submodules, so $\Omega(I)$ must be finite. Let $P$ be a nonzero prime ideal of $R$. $R / P \cong$ $\bigoplus \Sigma_{M \in \Omega} R_{M} / P_{M}$. If $M_{1}, M_{2} \in \Omega(P)$, then $R / P$ is decomposable and so $P$ is a finite intersection of properly larger ideals which is impossible since $P$ is a prime ideal. Thus, $\Omega(P)$ contains only one maximal ideal, and we have shown $R$ is $h$-local. Q.E.D.

It follows that the $h$-local integral domains are exactly the integral domains $R$ for which every nonzero ideal $I$ of $R$ we can find a decomposition $R / I \cong$ $\bigoplus \Sigma_{i=1}^{n} R / I_{i}$ with each $\Omega\left(I_{i}\right)$ containing exactly one maximal ideal.

Proposition 2. Suppose $I$ is an ideal of $R$. Then

(1) If $\Omega(I)$ is finite, then $R / I$ is a direct sum of indecomposable $R$-modules.

(2) If $R / I \cong \bigoplus \Sigma_{i=1}^{m} R / I_{i} \cong \bigoplus \Sigma_{j=1}^{n} R / J_{j}$ with $\Omega\left(I_{i}\right)=\Omega\left(J_{j}\right)$ for some $i$ and $j$, then for this $i$ and $j$ we have $I_{i}=J_{j}$.

(3) If $R$ is a Prüfer domain, then any two decompositions of $R / I$ as in (2) have a common finer decomposition $R / I \cong \bigoplus \Sigma_{i=1}^{m} \Sigma_{j=1}^{n} R / I_{i}+J_{j}$. 
Proof. (1) This follows from the fact that if $R / I \cong \bigoplus \Sigma_{i=1}^{m} R / I_{i}$, then $\left\{\Omega\left(I_{1}\right), \cdots, \Omega\left(I_{m}\right)\right\}$ is a partition of $\Omega(I)$.

(2) Suppose $R / I$ has the two given decompositions and $\Omega\left(I_{1}\right)=\Omega\left(J_{1}\right)$. Let $S=R-\bigcup \Omega\left(I_{1}\right) . S$ is a multiplicatively closed subset of $R$. Then $R / I_{1} \otimes_{R} R_{S}$ $\cong R / I_{1}$, and if $1<i \leqslant m$, then $I_{i} \cap S \neq \varnothing$ so $R / I_{i} \otimes_{R} R_{S} \cong\{0\}$. Thus, $R / I \otimes_{R}$ $R_{S} \cong R / I_{1}$. But $R / I \otimes_{R} R_{S}$ is independent of the decomposition of $R / I$, so $R / I_{1}$ $\cong R / J_{1}$ and this implies $I_{1}=J_{1}$.

(3) Suppose $R$ is a Prüfer domain and $R / I$ has the two given decompositions. We have $I=\bigcap_{i=1}^{m} I_{i},\left\{I_{1}, \cdots, I_{m}\right\}$ is pairwise comaximal, $I=\bigcap_{j=1}^{n} J_{j}$, and $\left\{J_{1}, \cdots, J_{n}\right\}$ is pairwise comaximal. Since $R$ is a Prüfer domain, the ideals of $R$ form a distributive lattice, i.e., $\bigcap_{j=1}^{n} I_{i}+J_{j}=I_{i}+\bigcap_{j=1}^{n} J_{j}$. Thus,

$$
\begin{aligned}
\bigcap_{i, j} I_{i}+J_{j} & =\bigcap_{i=1}^{m}\left(\bigcap_{j=1}^{n} I_{i}+J_{j}\right)=\bigcap_{i=1}^{m}\left(I_{i}+\bigcap_{j=1}^{n} J_{j}\right)=\bigcap_{i=1}^{m}\left(I_{i}+I\right) \\
& =\left(\bigcap_{i=1}^{m} I_{i}\right)+I=I .
\end{aligned}
$$

We have $\left\{I_{i}+J_{j}: i=1, \cdots, m\right.$ and $\left.j=1, \cdots, n\right\}$ is pairwise comaximal. Hence, the common finer decomposition exists by the Chinese Remainder Theorem. Q.E.D.

If $\Omega(I)$ is infinite with $I$ nonzero, then $R / I$ need not be a direct sum of indecomposable $R$-modules. By examples from number theory or either of the examples at the end of this paper, there exists an integral domain $R$ with appropriate maximal ideals $\left\{M_{i}\right\}_{i=1}^{\infty}$ such that $\bigcap_{i=1}^{\infty} M_{i} \neq\{0\}$ and $R / \bigcap_{i=1}^{\infty} M_{i}$ is not decomposable as a direct sum of indecomposable $R$-modules.

$R / I$ is indecomposable if $\Omega(I)$ contains a single maximal ideal. As a measure of the decomposability of $R / I$ when $\Omega(I)$ is finite, we have the following.

Proposition 3. Let $R$ be a Prïfer domain and $I$ an ideal of $R$ such that $\Omega(I)$ is finite. Then $R / I$ is indecomposable if and only if for all nontrivial partitions $\left\{\Omega_{1}, \Omega_{2}\right\}$ of $\Omega(I)$ there exist $M \in \Omega_{1}, N \in \Omega_{2}$ and a prime ideal $P$ of $R$ such that $I \subset P \subset M \cap N$.

Proof. Suppose $R / I$ is decomposable. Then $R / I \cong R / I_{1} \oplus R / I_{2}$ with $I_{1}$ and $I_{2}$ proper ideals of $R$. Then $I_{1} \cap I_{2}=I$. Suppose there exist $M \in \Omega\left(I_{1}\right)$, $N \in \Omega\left(I_{2}\right)$ and a prime ideal $P$ of $R$ such that $I \subset P \subset M \cap N$. $P \supset I$ implies $P \supset I_{1}$ or $P \supset I_{2}$. Suppose $P \supset I_{1}$. Then $N \in \Omega\left(I_{1}\right) \cap \Omega\left(I_{2}\right)$, contradicting $\left\{\Omega\left(I_{1}\right), \Omega\left(I_{2}\right)\right\}$ is a partition of $\Omega(I)$. Thus, it is not the case that such an $M, N$ and $P$ exist.

Conversely, suppose $R / I$ is indecomposable. Any $R$-submodule of $R / I$ is an

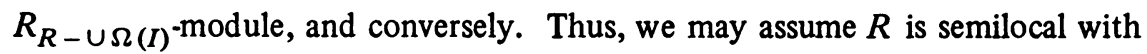


$\Omega=\Omega(I)$. Suppose $\left\{\Omega_{1}, \Omega_{2}\right\}$ is a nontrivial partition of $\Omega(I)$ and $\Omega_{1}=\left\{M_{1}\right.$, $\left.M_{2}, \cdots, M_{m}\right\}, \Omega_{2}=\left\{N_{1}, N_{2}, \cdots, N_{n}\right\}$. Let $v_{i}$ be the valuation associated with the valuation ring $R_{M_{i}}$, and $w_{j}$ be the valuation associated with the valuation ring $R_{N_{j}}$ (see [10]).

Suppose there exists $x_{1} \in R$ such that $v_{i}\left(x_{1}\right) \in v_{i}\left(I^{*}\right)$ and $w_{j}\left(x_{1}\right)=0$ for all $i$ and $j$, and suppose there exists $x_{2} \in R$ such that $v_{i}\left(x_{2}\right)=0$ and $w_{j}\left(x_{2}\right) \in$ $w_{j}\left(I^{*}\right)$ for all $i$ and $j$. Let $I_{1}=R x_{1}+I$ and $I_{2}=R x_{2}+I$. Then $I=I_{1} \cap I_{2}$ and $I_{1}+I_{2}=R$, so $R / I \cong R / I_{1} \oplus R / I_{2}$ and $R / I$ is decomposable. Contradiction. Thus, we may assume that there does not exist an $x \in R$ such that $v_{i}(x) \in v_{i}\left(I^{*}\right)$ and $w_{j}(x)=0$ for all $i$ and $j$.

For each $j$ suppose there exist $x_{j} \in R$ such that $v_{i}\left(x_{j}\right) \in v_{i}\left(I^{*}\right)$ for all $i$ and $w_{j}\left(x_{j}\right)=0 . R$ is a Bezout domain, since it is a semilocal Prüfer domain. Let $x \in R$ such that $R x=\sum_{j=1}^{n} R x_{j}$. We have $v_{i}(x) \in v_{i}\left(I^{*}\right)$ and $w_{j}(x)=0$ for all $i$ and $j$. Contradiction. Thus, there exists $j_{0} \in\{1,2, \cdots, n\}$ such that there does not exist $x \in R$ such that $v_{i}(x) \in v_{i}\left(I^{*}\right)$ for all $i$ and $w_{j_{0}}(x)=0$.

Suppose for each $i$ there exists $x_{i} \in R$ such that $v_{i}\left(x_{i}\right) \in v_{i}\left(I^{*}\right)$ and $w_{j_{0}}(x)$ $=0$. A finite intersection of principal ideals of $R$ is a principal ideal of $R$ since $R$ is a Bezout domain [1, Proposition 3.2]. Thus, there exists $x \in R$ such that $R x=$ $\bigcap_{i=1}^{m} R x_{i}$. Then $v_{i}(x) \in v_{i}\left(I^{*}\right)$ for all $i$ and $w_{j_{0}}(x)=0$. Contradiction. Thus, there exists $i_{0} \in\{1,2, \cdots, m\}$ such that there does not exist $x \in R$ such that $v_{i_{0}}(x) \in v_{i_{0}}\left(I^{*}\right)$ and $w_{j_{0}}(x)=0$.

As in [10] we define a subgroup $H$ of a totally ordered Abelian group $G$ to be an isolated subgroup if $H \neq G$ and if $g \in H$ whenever $g \in G$ and there exists $h \in H$ such that $-h \leqslant g \leqslant h$. Define $H=\{H: H$ is an isolated subgroup of $v_{i_{0}}\left(Q^{*}\right)$ and $\left.H \cap v_{i_{0}}\left(I^{*}\right)=\varnothing\right\}$. Let $H_{0}=\bigcup H$. Define $P$ by $P=\{0\} \cup\left\{r \in R^{*}\right.$ : $v_{i_{0}}(r) \notin H_{0}$ and $w_{j_{0}}(r)>0$. Clearly $P$ is an ideal of $R$ such that $I \subset P \subset M_{i_{0}} \cap$ $N_{j_{0}}$. We need to show that $P$ is a prime ideal of $R$.

First suppose there exists $r \in R^{*}$ such that $v_{i_{0}}(r) \notin H_{0}$ and $w_{j_{0}}(r)=0$. For every positive integer $p, v_{i_{0}}\left(r^{p}\right) \notin H_{0}$ and $w_{j_{0}}\left(r^{p}\right)=0$. By the earlier conclusion that there does not exist an $x \in R$ such that $v_{i_{0}}(x) \in v_{i_{0}}\left(I^{*}\right)$ and $w_{j_{0}}(x)=0$, we conclude that $v_{i_{0}}\left(r^{p}\right) \notin v_{i_{0}}\left(I^{*}\right)$ for all $p \geqslant 1$. Let $H_{1}=\left\{g \in v_{i_{0}}\left(Q^{*}\right)\right.$ : there exists $p \geqslant 1$ such that $\left.-v_{i_{0}}\left(r^{p}\right) \leqslant g \leqslant v_{i_{0}}\left(r^{p}\right)\right\}$. Then $H_{1} \in H$. But $v_{i_{0}}(r) \in$ $H_{1} \subset H_{0}$, contradiction. We have shown that there does not exist $r \in R$ such that $v_{i_{0}}(r) \notin H_{0}$ and $w_{j_{0}}(r)=0$.

To show that $P$ is a prime ideal, suppose $a, b \in R-P$. By the last paragraph $v_{i_{0}}(a) \in H_{0}$ and $v_{i_{0}}(b) \in H_{0}$. Thus, $v_{i_{0}}(a b)=v_{i_{0}}(a)+v_{i_{0}}(b) \in H_{0}+H_{0}$ $=H_{0}$, so $a b \notin P$, and $P$ is a prime ideal of $R$. Q.E.D. 
In particular, suppose $R$ is a Prüfer domain with $\Omega=\left\{M_{1}, M_{2}\right\}$ and $I$ an ideal of $R$ such that $I \subset M_{1} \cap M_{2}$. Then $R / I$ is indecomposable if and only if there exists a prime ideal $P$ of $R$ such that $I \subset P \subset M_{1} \cap M_{2}$.

Let $R$ be an integral domain. If $A$ is an $R$-submodule of $Q$, then we let $A^{-1}=\{q \in Q: q A \subset R\} . R$ is said to have a remote quotient field if there exists an $R$-submodule $A$ of $Q$ such that $A \neq Q$ and $A^{-1}=\{0\} . R$ is said to be a closed domain if $J \cap R \neq\{0\}$ for all nonzero ideals $J$ of $H$. Some facts concerning remote quotient fields and closed domains are given in [9] or [8].

THEOREM 4. Let $R$ be an integral domain. If $R$ does not have a remote quotient field, then $R$ is a closed domain.

Proof. The following argument was communicated by E. Matlis. By [9, Theorem 79] $R$ does not have a remote quotient field if and only if there exists a valuation ring $V$ such that $R \subset V \subset Q$ and $V^{-1} \neq\{0\}$. Thus, $V / R$ is an $R$-module which is torsion of bounded order. We get an exact sequence of $R$-modules

$$
\begin{array}{ccccc}
\operatorname{Hom}_{R}(K, V / R) \rightarrow \operatorname{Hom}_{R}(K, K) & \rightarrow \operatorname{Hom}_{R}(K, Q / V) \rightarrow \operatorname{Ext}_{R}^{1}(K, V / R) \rightarrow & \operatorname{Ext}_{R}^{1}(K, K) \\
\mathbb{R} & \mathbb{R} & \mathbb{R} & \mathbb{R} & \mathbb{R} \\
\{0\} & H & H(V) & V / R & \operatorname{Ext}_{R}^{1}(Q, R)
\end{array}
$$

where $H(V) \cong \operatorname{Hom}_{V}(Q / V, Q / V)$ is the completion of $V$ in the $V$-topology. Since $V / R$ is torsion (of bounded order) and $\operatorname{Ext}_{R}^{1}(Q, R)$ is torsion-free (and divisible), the last horizontal homomorphism is zero. Thus, we have a short exact sequence of $R$-modules $0 \rightarrow H \rightarrow H(V) \rightarrow V / R \rightarrow 0 . H \rightarrow H(V)$ is easily checked to be a ring homomorphism, so $H$ is an integral domain.

Let $Q_{H}$ and $Q_{H(V)}$ be the quotient fields of $H$ and $H(V)$, respectively. From the short exact sequence, we deduce that $Q_{H} \cong Q_{H(V)}$. Again from the short exact sequence and the fact that $H(V)$ is a valuation ring, we deduce that $H$ does not have a remote quotient field.

With identification we have $H \subset H \otimes_{R} Q \subset Q_{H}$. We have a short exact sequence $0 \rightarrow H \rightarrow H \otimes_{R} Q \rightarrow H \otimes_{R} K \rightarrow 0\left[9\right.$, p.9] and $H \otimes_{R} K \cong K[9$, Theorem 11], so $0 \rightarrow H \rightarrow H \otimes_{R} Q \rightarrow K \rightarrow 0$ is exact. If $h \in H^{*}$, then thinking of $h \in \operatorname{Hom}_{R}(K, K)$, we have $h(K) \neq\{0\}$. Thus, $\left\{h \in H: h\left(H \otimes_{R} Q\right) \subset H\right\}=\{0\}$. $H$ does not have a remote quotient field, then implies $H \otimes_{R} Q=Q_{H}$. If $h \in H^{*}$, then $1 / h \in Q_{H} \cong H_{R^{*}}$ and so there exist $h^{\prime} \in H$ and $r \in R^{*}$ such that $1 / h=h^{\prime} / r$. Thus, $r=h^{\prime} h \in(R \cap H h)^{*}$ and so every ideal $H h$ of $H$ intersects $R$ nontrivially. Q.E.D.

An integral domain is a Bezout domain if every finitely generated ideal is a principal ideal. Every Bezout domain is a Prüfer domain since finitely generated ideals, being principal, are projective. Every semilocal Prüfer domain is a Bezout 
domain [5, Corollary 5]. If $R$ is an integral domain and $A$ is an $R$-module, then $A$ is said to be $h$-reduced if $\operatorname{Hom}_{R}(Q, A)=\{0\}$. See [9] or [8] for some facts concerning the property $h$-reduced.

Lemma 5. Let $R$ be a Prïfer domain with exactly two maximal ideals $M_{1}$ and $M_{2}$. If $P$ is a prime ideal of $R$ such that $P \subset M_{1} \cap M_{2}$, then $P R_{P}=P$.

Proof. Straightforward (or see $[9$, proof of Theorem 54]).

THeOREM 6. Let $R$ be a Prüfer domain with exactly two maximal ideals. The following statements are equivalent:

(1) $R$ is not h-local.

(2) There exists a nonzero prime ideal of $R$ contained in both maximal ideals.

(3) $R$ does not have a remote quotient field.

(4) $V^{-1} \neq\{0\}$ for all valuation rings $V$ such that $R \subset V \subset Q$.

(5) There exists a valuation ring $V$ such that $R \subset V \subset Q$ and $V^{-1} \neq\{0\}$.

(6) Every proper $R$-submodule of $K$ is h-reduced.

(7) $K$ does not contain a nontrivial proper divisible submodule.

(8) Every nonzero element of $\operatorname{Hom}_{R}(K, K)$ is an epimorphism of $K$.

(9) $R$ is a closed domain.

(10) If $J$ is an ideal of $H$ and $I=J \cap R$, then $J=H I$.

(11) $H$ is an integral domain.

Proof. (1) $\Longleftrightarrow(2)$. Trivial.

(3) $\Rightarrow(2)$. [9, Theorem 94]

$(2) \Rightarrow(5)$. If $P$ is a nonzero prime ideal contained in both maximal ideals, then $R_{P}$ is a valuation ring and by Lemma $5, R_{P}^{-1} \supset P \neq\{0\}$.

(3) $\Leftrightarrow(4) \Leftrightarrow(5)$. [9, Theorem 79] .

(2) $\Rightarrow(7)$. Suppose $P$ is a nontrivial prime ideal of $R$ such that $P \subset M_{1} \cap$ $M_{2}$ where $M_{1}$ and $M_{2}$ are the two maximal ideals of $R$. Suppose $B / R$ is a divisible $R$-module with $Q \underset{\supsetneqq}{\supset} \underset{\supsetneqq}{\supset}$.

We wish to show that there exists $s \in R^{*}$ such that $1 / s+R \in(B / R)^{*}$. Let $q \in B-R$. If $q \notin R_{M_{1}} M_{1}$ and $q \notin R_{M_{2}} M_{2}$, then $1 / q \in R$ since $R_{M_{1}}$ and $R_{M_{2}}$ are valuation rings. Thus, $s=1 / q$ is the desired $s$. Otherwise we may without loss of generality assume that $q \notin R_{M_{1}}$ and $q \in R_{M_{2}} M_{2} . R$ is a Bezout domain, so there exists $g \in Q$ such that $R g=R+R q$. There exist $r_{1}, r_{2} \in R$ such that $g=r_{1}+r_{2} q$. Then $g+R=r_{1}+r_{2} q+R=r_{2}(q+R) \in B / R . g \notin R_{M_{1}}$ and $g \notin R_{M_{2}} M_{2}$, so $1 / g \in R_{M_{1}} \cap R_{M_{2}}=R$, and so $s=1 / g$ is the desired $s$. We have shown the existence of the required $s$.

We next show that there exists $t \in M_{1} \cap M_{2}$ such that $1 / t+R \in(B / R)^{*}$. 
Suppose this is not the case. We may assume without loss of generality that if $z \in R$ and $1 / z \in B-R$, then $z \notin M_{2}$. By the last paragraph, let $s \in R$ be chosen so that $1 / s+R \in(B / R)^{*}$. Choose $x \in P^{*}$. $B / R$ is a divisible $R$-module, so there exists $q \in B$ such that $1 / s+R=x(q+R)$. We claim that $1 / q \in R$. For if not $q \in R_{M_{i}} M_{i}$ for some $i=1$, 2. Then $x q \in P R_{M_{i}} M_{i}=R_{M_{i}} P \subset R_{P} P=P$ by Lemma 5. Then $1 / s+R=x q+R=0$, contradicting $1 / s+R \neq 0$. Thus, $1 / q$ $\in R$. Let $t=1 / q$, and so $1 / s+R=x(1 / t+R)$ with $1 / t+R \in B / R$. There exists $r \in R$ such that $1 / s-x / t=r$. If $r \in P$, then $t=x s+r s t \in P$ and this is the desired $t$. If $r \notin P$, then $t(1-r s)=x s \in P$. By the assumption that $1 / z \in$ $B-R$ implies $z \notin M_{2}$, we have $t \notin M_{2}$ and so $t \notin P$. Thus, $1-r s \in P \subset M_{1} \cap$ $M_{2}$ and so $r s$ and $s$ are units of $R .1 / s+R=0$, a contradiction. We have shown the existence of the required $t$.

Let $t \in M_{1} \cap M_{2}$ such that $1 / t+R \in(B / R)^{*} . B / R \neq K$ implies there exists $u \in R$ such that $1 / u+R \in K-B / R$. $B / R$ is divisible implies there exists $b \in B$ such that $1 / t+R=u(b+R)$. For some $r \in R$ we have $u b-1 / t=r$, or $b t=$ $(r t+1) / u$. $t \in M_{1} \cap M_{2}$ implies $r t+1$ is a unit of $R$, so $1 / u \in R b t \subset R b \subset B$, and so $1 / u+R \in B / R$ a contradiction.

(7) $\Rightarrow(8)$. Trivial.

(8) $\Rightarrow(11)$. Let $f, g \in \operatorname{Hom}_{R}(K, K)^{*}$. Then $f$ and $g$ are epimorphisms of $K$ and so $f g$ is an epimorphism of $K$. In particular $f g \neq 0$.

(11) $\Rightarrow$ (1). Suppose $R$ is $h$-local and $M_{1}$ and $M_{2}$ are the maximal ideals of $R$. Then $H \cong H\left(M_{1}\right) \oplus H\left(M_{2}\right)$ and so $H$ is not an integral domain.

(7) $\Rightarrow(6) \Rightarrow(8)$. Trivial.

(9) $\Rightarrow(10)$. [8, Proposition 7.8]

$(10) \Rightarrow(9) \Rightarrow(11)$. Trivial.

(3) $\Rightarrow(9)$. Theorem 4. Q.E.D.

If $R$ is an $h$-local integral domain, then we know the structure of $H$, namely $H \cong \Pi_{M \in \Omega} H(M)$ where $H(M)$ is the completion of $R_{M}$ in the $R_{M}$-topology. The following shows the possibility for $H$ when $R$ is not $h$-local.

Corollary 7. Let $R$ be a Prüfer domain with exactly two maximal ideals $M$ and $N$ and suppose $R$ is not h-local. Then $H$ is an integral domain with quotient field $H \otimes_{R} Q$ and $H \otimes_{R} Q / H \cong K$. The R-topology and H-topology on $H$ are the same, so $H$ is complete in the H-topology. $H$ is a Prüfer domain with exactly two maximal ideals $H M$ and $H N$, and $H$ is not h-local. Furthermore, $H=$ $H\left(R_{M}\right) \cap H\left(R_{N}\right), H\left(R_{M}\right)=H_{H M}$ and $H\left(R_{N}\right)=H_{H N}$ where we have identified these rings as subrings of the quotient field $H \otimes_{R} Q$ of $H$, and where $H\left(R_{M}\right)$ and $H\left(R_{N}\right)$ are the completions of $R_{M}$ and $R_{N}$, respectively.

Proof. By (11), $H$ is an integral domain, and in the proof of Theorem 4 we 
showed the quotient field of $H$ is $H \otimes_{R} Q$ and $H \otimes_{R} Q / H \cong K$. If $h \in H^{*}$, then there exists $r \in(R \cap H h)^{*}$ by (9). Thus, there exists $h^{\prime} \in H$ such that $r=h^{\prime} h$, and so $H r=H h^{\prime} h \subset H h$. Thus, the $R$-topology and the $H$-topology on $H$ are the same. $H$ is complete in the $R$-topology [9, Theorem 10], so $H$ is complete in the $H$-topology. $H$ is a flat $R$-module [9, Theorem 10], so by [2, Chapter VI, Proposition 4.1.2] $\operatorname{Tor}_{n}^{R}(A, C) \cong \operatorname{Tor}_{n}^{H}\left(A, H \otimes_{R} C\right)$ for every $H$-module $A$ and $R$ module $C$. By [3, Theorem 2] $R$ is a Prüfer domain if and only if $\mathrm{w} g l \operatorname{dim} R \leqslant 1$, i.e., $\operatorname{Tor}_{2}^{R}=0$. Hence, $\operatorname{Tor}_{2}^{H}=0$, and $H$ is a Prüfer domain. By [9, Theorem 13(2)], $H M$ and $H N$ are maximal ideals of $H$. By (10) there is a one-to-one correspondence between the ideals (prime ideals; maximal ideals) of $H$ and a subset of the set of ideals (respectively, prime ideals; maximal ideals) of $R$. Thus, $H$ has exactly these two maximal ideals. If $P$ is a nonzero prime ideal of $R$ such that $P \subset M \cap N$, then $H P$ is a prime ideal of $H[9$, Theorem 13(2)] and clearly $\{0\} \neq H P \subset H M \cap H N$, so $H$ is not $h$-local. Again using the proof of Theorem 4, we have $H \subset H\left(R_{M}\right) \subset$ $H \otimes_{R} Q$. If $J$ is an ideal of $H$, then by (10) $(R \cap J) H\left(R_{M}\right)=(R \cap J) H H\left(R_{M}\right)$ $=J H\left(R_{M}\right)$. Thus, $J \subset H M$ if and only if $R \cap J \subset M$ if and only if $(R \cap J) H\left(R_{M}\right)$ $\subset M H\left(R_{M}\right)$ if and only if $J H\left(R_{M}\right) \subset M H\left(R_{M}\right)$. Thus, $H_{H M}=H\left(R_{M}\right)$. Similarly $H_{H N}=H\left(R_{N}\right)$. Since $H$ is an integral domain $H=H_{H M} \cap H_{H N}=H\left(R_{M}\right) \cap$ $H\left(R_{N}\right)$. Q.E.D.

COROllary 8. Suppose $R$ is a Prüfer domain with exactly two maximal ideals. If $K$ is an injective $R$-module, then $R$ is h-local.

Proof. Suppose $K$ is an injective $R$-module and $R$ is not $h$-local. By (7) of the theorem, $K$ is indecomposable, and so by [7, Proposition 2.6] $H$ is a local ring. This contradicts Corollary 7. Q.E.D.

THEOREM 9. Let $R$ be an integral domain such that $M \not \subset \cup(\Omega-\{M\})$ for all $M \in \Omega$. If $K$ is an injective $R$-module, then $R$ is $h$-local.

Proof. Let $M \in \Omega$. By hypothesis, there exists $x_{M} \in M-\cup(\Omega-\{M\})$. Let $y_{M}=1 / x_{M}+R \in K$. $\operatorname{Ann}_{R}\left(y_{M}\right)=R x_{M}$. Let $E\left(R y_{M}\right)$ denote an injective envelope of $R y_{M}$. Since $K$ is injective, we can identify $E\left(R y_{M}\right)$ with a submodule of $K$, and we will denote this submodule by $K(M)$. We have $K(M)_{M} \neq\{0\}$ since $\left(R y_{M}\right)_{M} \cong\left(R / R x_{M}\right)_{M} \cong R_{M} / R_{M} x_{M} \neq\{0\}$. Let $C(M)$ be a complementary summand of $K(M)$ in $K$, i.e., $K=K(M) \oplus C(M) . K_{M} \cong Q / R_{M}$ which is indecomposable [9, Theorem 18]. On the other hand $K_{M} \cong K(M)_{M} \oplus C(M)_{M}$, so we must have $K(M)_{M} \cong Q / R_{M}$ and $C(M)_{M} \cong\{0\}$.

Let $N \in \Omega, N \neq M$. We claim that $K(M)_{N} \cong\{0\}$. Suppose not. By hypothesis, there exists $x_{N} \in N-\bigcup(\Omega-\{N\})$. Let $y_{N}=1 / x_{N}+R \in K$. Let $x=x_{M} x_{N}$ and $y=1 / x+R$. $(R y)_{P} \cong\{0\}$ if $P \in \Omega-\{M, N\}$ and $(R y)_{P} \neq\{0\}$ if $P \in$ 
$\{M, N\}$. Write $y=y_{1}+y_{2}$ where $y_{1} \in K(M)$ and $y_{2} \in C(M)$. As above, $K(M)_{N} \neq\{0\}$ implies $C(M)_{N} \cong\{0\}$. If $P \in \Omega-\{N\}$, then $\left(R y_{2}\right)_{P} \cong\{0\}$. Thus, $\left(R y_{2}\right)_{P} \cong\{0\}$ for all $P \in \Omega$, and so $y_{2}=0$. Hence, $y \in K(M)$. $x_{M} y=y_{N} \in$ $K(M)^{*}$, and $R y_{N} \cap R y_{M}=\{0\}$. But this is impossible since $K(M)$ is an essential extension of $R y_{M}$. We have shown that $K(M)_{N} \cong\{0\}$ if $N \in \Omega, N \neq M$.

For each $M \in \Omega$ we get a submodule $K(M)$ of $K$. We wish to show that $K$ is the direct sum of these submodules. For a given $M \in \Omega$, let $A=K(M) \cap$ $\Sigma_{P \in \Omega-\{M\}} K(P)$. For $P \in \Omega, P \neq M, A_{P} \neq\{0\}$ since $K(M)_{P} \cong\{0\}$. On the other hand

$$
A_{M} \subset\left(\sum_{P \in \Omega-\{M\}} K(P)\right)_{M} \cong\{0\}
$$

since $K(P)_{M} \cong\{0\}$. Thus, $A_{N} \cong\{0\}$ for all $N \in \Omega$, and so $A \cong\{0\}$. The sum $\Sigma_{P \in \Omega} K(P)$ is therefore a direct sum. If $f: \bigoplus \Sigma_{P \in \Omega} K(P) \rightarrow K$ is the inclusion map, $M \in \Omega$, and $1_{M}: R_{M} \rightarrow R_{M}$ the identity map, then the induced map

$$
f \otimes 1_{M}:\left(\bigoplus \sum_{P \in \Omega} K(P)\right) \otimes_{R} R_{M} \rightarrow K \otimes_{R} R_{M}
$$

is an isomorphism. It follows that $f$ is an isomorphism, i.e., $K=\bigoplus \Sigma_{P \in \Omega} K(P)$.

Let $r \in R^{*}$. There exist $P_{1}, \cdots, P_{n} \in \Omega$ such that $1 / r+R=k_{1}+\cdots+$ $k_{n}$ where $k_{i} \in K\left(P_{i}\right)$. Thus, if $P \in \Omega-\left\{P_{1}, \cdots, P_{n}\right\}$ then $(R(1 / r+R))_{P} \cong\{0\}$, and so $\Omega(r) \subset\left\{P_{1}, \cdots, P_{n}\right\}$, a finite set. Thus, if $R$ is not $h$-local, then there exists a nonzero prime ideal $J$ of $R$ contained in more than one maximal ideal of $R$. Let $j \in J^{*}$. There exist $P_{1}, \cdots, P_{n} \in \Omega$ such that $1 / j+R=j_{1}+\cdots+j_{n}$ where $j_{i} \in K\left(P_{i}\right)$. Then

$$
J \supset R j=\operatorname{Ann}_{R}(1 / j+R)=\bigcap_{i=1}^{n} \operatorname{Ann}_{R}\left(j_{i}\right) .
$$

$J$ is a prime ideal implies there exists an $i$ such that $J \supset \operatorname{Ann}_{R}\left(j_{i}\right)$. If $M, N \in \Omega$ and $M \supset J, N \supset J$, and for this $i$, we have $\left(R j_{i}\right)_{M} \neq\{0\}$ and $\left(R j_{i}\right)_{N} \neq\{0\}$. Hence, $K\left(P_{i}\right)_{M} \neq\{0\}$ and $K\left(P_{i}\right)_{N} \neq\{0\}$, contradicting the second paragraph. Thus, $R$ must be $h$-local. Q.E.D.

It is not known whether the hypothesis of "every maximal ideal is not contained in the union of the other maximal ideals" is necessary for Theorem 9. It is an interesting open question as to which integral domains have the property that $K$ is an injective $R$-module. Especially in light of the fact that for a valuation ring $R$ we have that $K$ is an injective $R$-module if and only if every finitely generated $R$-module is a direct sum of cyclic submodules, or more generally this is true for semilocal $h$-local Prüfer domains [1, Theorem 4.10]. The following generalizes Corollary 8. 
COROLlaRY 10. Let $R$ be a semilocal integral domain. If $K$ is an injective $R$-module, then $R$ is $h$-local.

Proof. If $R$ is semilocal, then no maximal ideal of $R$ is contained in the union of the rest of the maximal ideals of $R$. Q.E.D.

In [1] we define a ring $R$ to be almost maximal if every proper homomorphic image of $R$ is a linearly compact $R$-module. Then $R$ is an almost maximal integral domain if and only if $R$ is an $h$-local integral domain and $R_{M}$ is almost maximal for all $M \in \Omega$ [1, Theorem 2.9]. The following generalizes Theorem 54 of [9] and provides one of the missing implications of Theorem 4.8 of [1].

Theorem 11. Let $R$ be a semilocal Prïfer domain. The following statements are equivalent:

1. $K$ is an injective $R$-module.

2. $R$ is an almost maximal integral domain.

Proof. Suppose $K$ is an injective $R$-module. By Corollary 10, $R$ is $h$-local, and so $R$ is almost maximal by [1, Theorem 4.9]. The converse follows from [1, Theorem 4.8]. Q.E.D.

THEOREM 12. Let $R$ be a Bezout domain such that $M \not \subset \bigcup(\Omega-\{M\})$ for all $M \in \Omega$ and such that $K$ is an injective $R$-module. Then every finitely generated $R$-module is a direct sum of cyclic submodules.

Proof. By Theorem $9, R$ is $h$-local, so $R$ is almost maximal by [1, Theorem 4.9]. An almost maximal Bezout domain has the property that every finitely generated module is a direct sum of cyclic submodules [1, Theorem 3.4]. Q.E.D.

COROllary 13. Let $R$ be a semilocal Prüfer domain with $K$ an injective $R$ module. Then every finitely generated $R$-module is a direct sum of cyclic submodules.

Proof. A semilocal Prüfer domain is a Bezout domain, and no maximal ideal of $R$ is contained in the union of the rest of the maximal ideals of $R$ since $R$ is semilocal. Q.E.D.

We now turn our attention to the hypothesis of Theorems 9 and 12. If $X$ is a set, $F$ is a filter of $X$ if (1) $\varnothing \neq F \subset X$ for all $F \in F$, (2) $F_{1} \cap F_{2} \in F$ for all $F_{1}, F_{2} \in F$, and (3) $F \in F$ and $F \subset F^{\prime} \subset X$ implies $F^{\prime} \in F$. $F$ is an ultrafilter of $X$ if $F$ is a filter of $X$ and is maximal with respect to that property. By Zorn's Lemma every filter of $X$ is contained in an ultrafilter of $X$. F is a free filter (or ultrafilter) of $X$ if $\cap F=\varnothing$.

Proposition 14. Let $R$ be a Bezout domain with $M \in \Omega$ such that $M \subset$ $\bigcup \Omega^{\prime}$ where $\Omega^{\prime}=\Omega-\{M\}$. For $x \in R$ define $\Omega^{\prime}(x)=\left\{P \in \Omega^{\prime}: x \in P\right\}$. Then 
there exists a free ultrafilter $F$ of $\Omega^{\prime}$ such that $M=\left\{x \in R: \Omega^{\prime}(x) \in F\right\}$.

Proof. Let $F_{0}=\left\{\Omega^{\prime}(x): x \in M\right\}$. If $x_{1}, \cdots, x_{n} \in M$, then since $R$ is a Bezout domain, there exists $g \in M$ such that $R g=R x_{1}+\cdots+R x_{n}$. Thus, $\Omega^{\prime}(g) \subset \bigcap_{i=1}^{n} \Omega^{\prime}\left(x_{i}\right) . M \subset \cup \Omega^{\prime}$ implies $\Omega^{\prime}(g) \neq \varnothing$. We have that if $F_{1}, \cdots$, $F_{n} \in F_{0}$, then $\bigcap_{i=1}^{n} F_{i} \neq \varnothing$. Let $F_{1}=\left\{F \subset \Omega^{\prime}:\right.$ there exist $F_{1}, \cdots, F_{n} \in F_{0}$ such that $F \supset \bigcap_{i=1}^{n} F_{i}$ \}. Then $F_{1}$ is a filter of $\Omega^{\prime}$. Let $F$ be an ultrafilter of $\Omega^{\prime}$ containing $F_{1}$. If $P \in \Omega^{\prime}$, then $P \not \supset M$, so there exists $x \in M-P$. Then $P \notin$ $\Omega^{\prime}(x)$, and so $\bigcap F_{0}=\varnothing$. Hence, $F$ is a free ultrafilter.

Let $M^{\prime}=\left\{x \in R: \Omega^{\prime}(x) \in F\right\}$. If $x \in M^{\prime}$ and $r \in R$, then $\Omega^{\prime}(r x) \supset \Omega^{\prime}(x)$ $\in F$ and so $r x \in M^{\prime}$. If $x, y \in M^{\prime}$, then $\Omega^{\prime}(x+y) \supset \Omega^{\prime}(x) \cap \Omega^{\prime}(y) \in F$, and so $x+$ $y \in M^{\prime}$. Thus $M^{\prime}$ is an ideal of $R$. $\Omega^{\prime}(1)=\varnothing \notin F$, so $M^{\prime} \neq R$. Clearly $M^{\prime} \supset M$, and so $M^{\prime}=M$. Q.E.D.

COROllary 15. Let $R$ be a Bezout domain with $M \in \Omega$ such that $M \subset$ $\cup(\Omega-\{M\})$. Then $R$ is not h-local.

Proof. If $F$ is a free filter and $F \in F$, then $F$ is an infinite set. Hence, every element of $M$ is contained in infinitely many maximal ideals, so $R$ is not $h$ local by definition. Q.E.D.

COROllary 16. Let $R$ be a Bezout domain such that $K$ is an injective $R$ module. Then $R$ is h-local if and only if for every $M \in \Omega$ we have $M \not \subset$ $\bigcup(\Omega-\{M\})$.

Proof. Combine Theorem 9 and Corollary 15.

The following two examples seem to indicate that the property of a maximal ideal being contained in the union of other maximal ideals is not related to the Krull dimension of the ring. Namely, there exist two Bezout domains each with maximal ideals $M_{\infty}, M_{1}, M_{2}, \cdots$ such that $M_{\infty} \subset \bigcup_{n=1}^{\infty} M_{n}$ and the Krull dimension of $R_{M_{n}}$ is one for all positive integers $n$, yet the Krull dimension of $R_{M_{\infty}}$ is one for the one ring and infinity for the other ring.

EXAMPLE 17. If $R$ is an integral domain with $U$ the set of units of $R$, then the Abelian group $Q^{*} / U$ is called the divisibility group of $R$. It is a partially ordered group where $a U \geqslant b U$ for $a, b \in Q^{*}$ if $a / b \in R$. P. Jaffard has proved that given any lattice ordered Abelian group $G$ there exists an integral domain with divisibility group $G[6$, Theorem 3, p. 78$]$. In fact the integral domain thus constructed is a Bezout domain $[4$, p. 1370]. Let $N=\{1,2, \cdots\}$ be the set of natural numbers, and let $Z$ be the additive group of integers. $Z^{N}$ is then a countable product of copies of $Z$, and we can think of an element of $Z^{N}$ of the form $\left(z_{1}\right.$, $\left.z_{2}, \cdots\right)$ or simply $\left(z_{n}\right)$ where $z_{n} \in Z$. Let $G=\left\{\left(z_{n}\right) \in Z^{N}\right.$ : there exists $k \in Z$ 
such that $z_{n}=k$ except for a finite number of $\left.n \in N\right\}$. Order $G$ by $\left(z_{n}\right) \geqslant\left(z_{n}^{\prime}\right)$ if $z_{n} \geqslant z_{n}^{\prime}$ for all $n \in N$. Then $G$ is a lattice ordered group. By the Jaffard construction, there exists a Bezout domain $R$ with divisibility group $G$. Let $f$ be the composition $Q^{*} \rightarrow Q^{*} / U \cong G$. For $i \in N$ let $M_{i}=\{0\} \cup\left\{r \in R^{*}:\right.$ if $f(r)=\left(z_{n}\right)$, then $z_{n} \geqslant 0$ for all $n \in N$ and $\left.z_{i}>0\right\}$. Let $M_{\infty}=\{0\} \cup\left\{r \in R^{*}:\right.$ if $f(r)=\left(z_{n}\right)$, then $z_{n} \geqslant 0$ for all $n \in N$ and $z_{n}>0$ for all but a finite number of $\left.n \in N\right\}$. It is not hard to see that $\Omega=\left\{M_{\infty}, M_{1}, M_{2}, \cdots\right\}$ and if $M \in \Omega$, then $R_{M}$ is a discrete rank one valuation ring. Thus, the Krull dimension of $R_{M}$ is one for all $M \in \Omega$. Clearly $M_{\infty} \subset \bigcup_{n \in N} M_{n}$. Directly or using Corollary 15 , one sees that $R$ is not $h$-local. (This is an example of a locally Noetherian ring which is not Noetherian, appearing in [W. Heinzer and J. Ohm, Locally Noetherian commutative rings, Trans. Amer. Math. Soc. 158 (1971), 273-284].)

EXAMPLE 18. Use the same notation as in the last example, but think of replacing $G$ by $Z^{N}$. Use the Jaffard construction to get a Bezout domain $R$ with divisibility group $Z^{N}$. With the corresponding $M_{n}$, there exists a maximal ideal $M_{\infty}$ of $R$ such that $M_{\infty} \subset \bigcup_{n \in N} M_{n}$ (see Proposition 14 for construction of $M_{\infty}$ ). It car be shown that for $n \in N, R_{M_{n}}$ is a discrete rank one valuation ring and hence $R_{M_{n}}$ is of Krull dimension one. With a little more difficulty, it can be shown that the Krull dimension of $R_{M_{\infty}}$ is infinity. Again $R$ is not $h$-local by Corollary 15 .

\section{BIBLIOGRAPHY}

1. W. Brandal, Almost maximal integral domains and finitely generated modules, Trans. Amer. Math. Soc. 183 (1973), 203-222.

2. H. Cartan and S. Eilenberg, Homological algebra, Princeton Univ. Press, Princeton, N. J., 1956. MR 17, 1040.

3. A. Hattori, On Prüfer rings, J. Math. Soc. Japan 9 (1957), 381-385. MR 20 \#854.

4. W. Heinzer, J.noetherian integral domains with 1 in the stable range, Proc. Amer. Math. Soc. 19 (1968), 1369-1372. MR38 \#145.

5. Y. Hinohara, Projective modules over semilocal rings, Tôhoku Math. J. (2) 14 (1962), 205-211. MR 31 \#4814.

6. P. Jaffard, Les systemes d'idéaux, Travaux et Recherches Math., IV, Dunod, Paris, 1960. MR 22 \#5628.

7. E. Matlis, Injective modules over Noetherian rings, Pacific J. Math. 8 (1958), 511 528. MR 20 \#5800.

8. - Cotorsion modules, Mem. Amer. Math. Soc. No. 49 (1964). MR 31 \#2283.

9. - Torsion-free modules, Univ. of Chicago Press, Chicago, Ill., 1972.

10. O. Zariski and P. Samuel, Commutative algebra. Vol. II, University Series in Higher Math., Van Nostrand, Princeton, N. J., 1960. MR 22 \#11006.

DEPARTMENT OF MATHEMATICS, COLORADO STATE UNIVERSITY, FORT COLLINS, COLORADO 80521 